\title{
Intervención psicológica en un caso de infertilidad femenina Infertility female intervention
}

\author{
Ana $\mathrm{M}^{\mathrm{a}}$ Regueiro Ávila ${ }^{1,2}$ y Luís Valero Aguayo ${ }^{2}$ \\ ${ }^{1}$ Servicio de Atención Psicológica, Universidad de Málaga, España. \\ ${ }^{2}$ Departamento de Personalidad, Evaluación y Tratamiento Psicológico, Universidad de Málaga, España.
}

Disponible online 31 de diciembre de 2011

Se describe el caso clínico de una mujer de 30 años con problemas de ansiedad y depresión provocados por un problema de infertilidad y varios intentos de tratamientos de reproducción asistida sin éxito. Se ha realizado un diseño de caso único con una evaluación pre-post-seguimiento. Tras un análisis funcional se diseñó un programa de tratamiento cognitivo-conductual con multicomponentes. Se aplicó durante 15 sesiones e incluyó: biblioterapia sobre infertilidad, relajación, tareas gratificantes, expresión de sentimientos, entrenamiento en solución de problemas y toma de decisiones, ejercicios de visualización y reestructuración cognitiva. Los resultados mostraron una mejora progresiva de su estado de ánimo y una disminución de los niveles de ansiedad, con criterios de normalidad al final del tratamiento. En la última sesión de tratamiento la paciente tomó la decisión de emprender un último intento mediante la donación de ovocitos, antes de considerar la posibilidad de adopción. Se realizó un seguimiento al mes, donde habían disminuido aún más los niveles de ansiedad y depresión, e informó que se había quedado embarazada, dando a luz posteriormente a dos niñas completamente sanas. Se realizaron seguimientos telefónicos a los 3, 6 y 12 meses que confirmaron el mantenimiento de estos beneficios.

Palabras clave: Infertilidad Femenina; Intervención Psicológica; Cognitivo-conductual; Ansiedad; Depresión.

This article describes the clinical case of a 30-year-old woman suffering with anxiety and depression problems provoked by an infertility problem and several unsuccessful attempts of assisted reproduction therapy. The study followed a single-case design with pre/post/follow-up assessment. After a functional analysis a cognitive-behavioral treatment program was designed with multicomponent variables. It was applied for 15 sessions and included: literature on infertility, relaxation training, gratifying tasks, managing feelings, training in problem solving and making decisions, visualization exercises and cognitive restructuring. The results showed progressive improvement in her mood and a gradual decrease in the levels of anxiety over the treatment period, with she fulfilled criteria for normality at the end-of-treatment. In the last session, the patient decided to have one final attempt at pregnancy through oocyte donation, before considering the possibility of adoption. A follow-up clinical session was established 1-month later showing that the anxiety and depression levels had decreased and that the patient was pregnant. She experienced a totally normal pregnancy and delivered 2 completely healthy girls. Follow-up phone calls were made at 3, 6 and 13 months confirmed that the benefits had been retained.

Keywords: Female Infertility; Psychological Treatment; Cognitive-behavioral; Anxiety; Depression.

Correspondencia: Ana Ma Regueiro Ávila. Facultad de Psicología. Campus Teatinos, 29071 Málaga. E-mail: amregueiro@uma.es. E-mail de los otros autores: Luís Valero: 1valero@uma.es. 
En la actualidad, existe un alto porcentaje de infertilidad en la población occidental debido a una multitud de factores tales como: diferenciación entre los conceptos placer y reproducción, aumento en el empleo de las técnicas de control de la natalidad, retraso en la edad, tanto en la mujer como en el hombre para tomar la decisión de tener hijos (con el consiguiente envejecimiento de los gametos y la disminución de la calidad seminal), incorporación de la mujer al mundo laboral, cambio en los roles sexuales y estilo de vida, el aumento de las enfermedades de transmisión sexual, y la precocidad en el inicio de las relaciones (World Health Organization, 2003). La consecuencia de todos estos factores es una falta del reemplazo generacional por los bajos índices de natalidad. En España la tasa de natalidad ha ido descendiendo en los últimos años, desde un 18.76 en 1976 hasta el 10.75 por cada 1000 habitantes en 2009 , lo que supone una media de 1.38 hijos por mujer (Instituto Nacional de Estadística, 2010). Estos índices fueron disminuyendo a lo largo la década de los 80 y 90 , pero remontaron posteriormente gracias al fenómeno de la inmigración.

Aunque en la literatura aparece cierta confusión en cuanto a los términos infertilidad y esterilidad (a veces incluso se utilizan como sinónimos), se adopta aquí la definición de Daya (1998) sobre "infertilidad" como la incapacidad para engendrar hijos tras un periodo prolongado, de uno a dos años, manteniendo relaciones sexuales sin protección; mientras que "esterilidad" se entiende como la imposibilidad absoluta para concebir. Actualmente existe un acuerdo generalizado en los especialistas sobre el tema en el hecho de que procesos de estrés, ansiedad y depresión pueden contribuir a la infertilidad funcional, y también a la inversa un problema de infertilidad fisiológica puede llevar a graves problemas de ansiedad y depresión, y problemas de pareja (Antequera, Moreno-Rosset, Jenaro y Avila, 2008; Llavona, 2008; Seger, 2003).

La mayoría de las parejas infértiles (entre el 25 y el $65 \%$ según varios autores) consideran la infertilidad y su tratamiento como una de las experiencias más estresantes y dolorosas de su vida. Se estima que entre el 59.60 y el $67 \%$ de las mujeres remitidas a servicios de infertilidad presentan también problemas de ansiedad y alguna comorbilidad psiquiátrica. Asimismo, dichos problemas aumentan cuanto mayor es el número de ciclos de tratamiento de infertilidad, especialmente en momentos clave de las técnicas de reproducción asistida, como la extracción de óvulos y la transferencia de embriones (Guerra, 1998, 2000). Domar (1997) describe varios estudios que indican que las mujeres infértiles tienen índices altos de ansiedad y depresión, y que se ven más afectadas que sus parejas en este sentido (Miranda, Larrazabal y Laba, 1995; Moreno-Rosset, 2000; Newton, Hearn y Yuzpe, 1990). Otros autores informan que las mujeres con infertilidad padecen niveles de depresión y ansiedad equivalentes a otros problemas como sida, cáncer o alteraciones cardiovasculares (Castro, Borrás, Pérez-Pareja y Palmer, 2001; Domar, Zuttermeister y Friedman, 1993; Palacios, Jadresic, Palacios, Miranda y Domínguez, 2002).
También hay estudios que informan que todos estos problemas se asocian con una menor posibilidad y porcentaje en concebir (Demyttenaere, Nijs, Evers-Kiebooms y Koninc, 1991; Domar, 1997; Guerra y Veiga, 1994; Moreno-Rosset, 2000; Newman y Zouves, 1991; Tarlatzis et al., 1993). En este tipo de estudios se tiende a afirmar que el estrés puede producir una menor probabilidad de conseguir el embarazo y una mayor posibilidad de aborto espontáneo en mujeres que presentan problemas emocionales o trastornos psicológicos. De hecho, las situaciones crónicas y mantenidas de estrés afectan al sistema endocrino, la pituitaria segrega prolactina y ésta inhibe la actividad reproductora, disminuye la cantidad de estrógenos y se produce una menor probabilidad de ovulación, la progesterona interrumpe también la maduración de las paredes uterinas dificultando incluso el probable implante del embrión (Robles y Peralta, 2006). En la mayoría de las parejas infértiles con etiología desconocida es muy probable que los factores psicológicos jueguen un papel muy importante (Guerra, Llobera, Veiga y Barri, 1998; Guerra et al., 2009; Llavona y Mora, 2003; Moreno-Rosset, 2007). De hecho, en las unidades de reproducción asistida existen algunos servicios psicológicos y la demanda continúa aumentando (Moreno-Rosset, Jenaro, Antequera y Gómez, 2009).

La reducción de estos síntomas aumenta el número de concepciones y mejora la integración de los pacientes en los programas de reproducción asistida. Una ayuda psicoterapéutica puede ayudar a tener más éxito en los tratamientos de reproducción asistida, disminuyendo el estrés y la depresión y también modificando las actitudes. Los porcentajes de éxito aumentan en aquellos tratamientos de parejas que han aprendido a manejar correctamente su problemática (Domar et al., 2000; Sanders y Bruce, 1999). Cuando la adaptación personal al proceso mejora, aumentan las posibilidades de ovulación, fecundación, nidación de los embriones y consecución del embarazo. Cuanto más activos y positivos sean los miembros de la pareja y cuanto más perciban tener una mayor sensación de control sobre su problema y su tratamiento, mayores serán las posibilidades de concepción.

Algunas experiencias han demostrado que aquellas parejas que tienen un mayor ajuste personal, marital y social, son los que mejor se integran en los programas de reproducción asistida, mejores resultados obtienen y mayor satisfacción expresan. El apoyo psicológico hace que estas parejas estén más satisfechas con los tratamientos de reproducción asistida $\mathrm{y}$, por tanto, decidan aumentar el número de ciclos (Guerra y Veiga, 1994).

Entre los programas estructurados para este tipo de tratamientos se encuentra el Programa Mente-Cuerpo realizado por la Dra. Alice D. Domar y colaboradores en el Beth Israel Deaconmes Medical Center de Boston (Domar, Seibel y Benson, 1990). Es un programa de corte cognitivo-conductual estructurado a lo largo de 10 sesiones de dos horas de duración. Las pacientes son mujeres que se encuentran en cualquier 
fase del proceso de infertilidad, sus parejas pueden asistir a dos sesiones. Las técnicas que se utilizan son la relajación, el ejercicio físico, el incremento de emociones positivas, la reestructuración cognitiva, la expresión de emociones, estrategias para expresar y manejar los temores, yoga, reducción de estrés a través del humor o potenciar la autoafirmación. Dicha autora afirma que las mujeres de dicho programa lograron quedarse embarazadas antes y en un mayor porcentaje que aquéllas que sólo recibieron el tratamiento médico correspondiente (un 36\% de partos, incluso un $57 \%$ de embarazos en mujeres deprimidas).

Otras experiencias terapéuticas destacables son las de Tuschen, Florín, Krause y Pook (1999) y las de Dolz y García (2002). Éstos últimos realizan un Programa de Apoyo Psicológico, dirigido a parejas infértiles que consta de 8 sesiones y se centra en aspectos como: dar información específica, técnicas de relajación, autocontrol y entrenamiento en habilidades personales. Los datos que han publicado informan de una tasa de embarazos del 54\% frente a un 28\% del grupo control, también hallaron diferencias significativas en cuanto a la tasa de abandono de los tratamientos $(5.70 \%$ frente al $37.50 \%$ del grupo control).

Los programas de intervención psicológica deberían incluir técnicas cognitivo- conductuales para el tratamiento de la ansiedad y la depresión, así como el establecimiento de estrategias de afrontamiento ante todas las fases que atraviesan las parejas infértiles (Ávila y Moreno-Rosset, 2008; Flores, Jenaro y Moreno, 2008). Estas fases son: el diagnóstico de la infertilidad, la información acerca de los programas de reproducción asistida, la toma de decisiones respecto al programa de reproducción concreto que va a iniciar la pareja y la aplicación del programa en sí mismo (tratamiento farmacológico, aplicación del procedimiento, espera de resultados y resultado final). Los profesionales implicados en la reproducción asistida deberían ser conscientes de la importancia de los factores psicológicos en los resultados de los tratamientos de fertilidad.

Se presenta aquí, pues, un caso clínico como muestra de la eficacia de la intervención psicológica en el proceso de tratamiento de reproducción asistida, mostrando el proceso terapéutico seguido, en las distintas fases sucesivas, los datos de eficacia y la consecución final de la reproducción con éxito total.

\section{Descripción del caso}

Milagros era una estudiante de 30 años que acude a consulta del Servicio de Atención Psicológica de la Universidad porque decía padecer un estado de ánimo depresivo y numerosos problemas de ansiedad a raíz del diagnóstico de infertilidad y tras varios intentos de procedimientos de reproducción asistida sin éxito. Ella misma se definía como "muy ansiosa desde siempre", pero no había acudido anteriormente a ningún profesional ni había tenido tratamientos psiquiátricos o psicológicos anteriores. A partir de las entrevistas de evaluación iniciales se extrajo la siguiente información: Milagros y su pareja habían iniciado sus intentos de concebir un hijo hacía tres años: después de un año, al ver que no se producía el embarazo, deciden acudir a los profesionales médicos pertinentes e iniciaron las pruebas médicas oportunas para averiguar el origen del problema. Estas pruebas manifestaron un factor de infertilidad femenina: Milagros padecía ciertas anomalías en las trompas de Falopio y sus ovocitos eran de mala calidad. En ese primer momento y tras el diagnóstico, Milagros pasa por una serie de fases muy parecidas a las de un duelo, pues se puede considerar así la pérdida en un proceso de fecundación de un hijo deseado: a) Fase de shock, con gran impacto y dolor emocional por su proyecto de familia frustrado (ambos provienen de familias numerosas y todos sus hermanos tienen varios hijos); b) Fase de negación, con afirmaciones constantes como " $n o$ puede ser", "es un error"; c) Fase de culpabilización hacia sí misma o su pareja, con frases como "es por culpa mía, por mi naturaleza", "es porque él ha tenido otras relaciones antes"; d) Ansiedad, con dudas constantes como "¿es que nunca voy a quedarme embarazada?"; e) Depresión, con duelo por el hijo no nacido y por la pérdida de un cierto proyecto de vida, con afirmaciones del tipo "no tengo nada"; y también f) Baja autoestima con afirmaciones como "no soy nada", "no valgo nada", "soy inferior".

La pareja toma la decisión de iniciar los programas de reproducción asistida en centros privados, con el consiguiente coste económico para ellos, por creer que la atención privada ofrecería un mejor servicio y una mayor probabilidad de éxito. Realizan por tanto cinco intentos seguidos (dos inseminaciones, dos fecundaciones in vitro y una microinyección intracitoplasmática) a lo largo de dos años. En ninguno de ellos consiguió una fecundación positiva. En una clínica especializada le informaron como hipótesis de un problema genético que impedía el embarazo, sin especificar cuál. Esta situación continua de médicos, análisis e intentos sucesivos, se convirtió para Milagros en una situación altamente estresante, crónica y mantenida a lo largo de esos años. Los problemas (conductuales y emocionales) que presentaba Milagros tras los sucesivos fracasos pueden resumirse en los siguientes: a) cambios emocionales fuertes, con subidas y bajadas según los ciclos y periodos de tratamientos de infertilidad, a veces con ilusión y otras con decepción por el fracaso, cuantos más intentos realizaban mayor la sensación de desesperanza; b) pensamientos sobre pérdida de control, imposibilidad de hacer nada para solucionar sus problemas e indefensión, con afirmaciones del tipo "no puedo hacer nada", "no puedo controlar esta situación"; c) emociones de rabia hacia sí misma y envidia hacia otras parejas con hijos, decía cosas como "¿por qué yo no y ellos sí?”; d) sensaciones de soledad y aislamiento, con renuncia a actividades sociales y afirmaciones del tipo "nadie me puede entender", "no me apetece nada"; e) problemas de comunicación e interacción con otras personas cercanas, le costaba expresar sus emociones a su propia pareja, incluso ocultaba la situación a los familiares; y f) 
problemas sexuales, con bajo deseo y dificultades para llegar al orgasmo en sus relaciones.

La pareja de Milagros, aunque al principio también le afectó esta situación, había asumido el problema de manera más adaptativa. En el momento de la evaluación tenía una labor de apoyo emocional hacia Milagros y la secundaba en las decisiones que ella iba tomando, también era quien pagaba todos los costes económicos de los procesos médicos. La relación de pareja era muy buena, pero por su trabajo y horarios no podía acudir a nuestras sesiones. Por este motivo el proceso terapéutico se centró exclusivamente en Milagros, aunque se realizaron algunos contactos telefónicos con él para darle algunas indicaciones y recomendaciones concretas. En el momento de acudir a consulta, Milagros estaba a la espera de comenzar su sexto intento tras un periodo de 6 meses de descanso hormonal. Según la descripción anterior, presentaba pues las características para un diagnóstico de trastorno del estado de ánimo y ansiedad debido a enfermedad médica (F06.32 y F06.4, American Psychiatric Association, 2002). No correspondería un diagnóstico de "trastorno adaptativo" debido a la larga historia de más de tres años con estos problemas y tener características similares a un problema depresivo y una reacción de duelo.

\section{Evaluación}

\section{Método}

Se realizaron 3 sesiones de evaluación en las que se utilizaron diferentes entrevistas, concretamente una primera entrevista conductual centrada en el problema y una entrevista semiestructurada del propio Servicio de Atención Psicológica. En esas entrevistas, además del historial clínico y características del problema, se registraban también frases literales como indicadores de los pensamientos y estado emocional de Milagros durante las sesiones. Como parte del protocolo de este servicio se le solicitaba también autorización por escrito para recoger sus datos y su posible publicación. Las informaciones personales que pudieran servir de identificación, que aparecen aquí descritas, no son reales. Se recogió la información médica y la farmacología actual. Se le aplicó el Inventario de Depresión de Beck (Beck, Ward y Mendelson, 1961/1975), en el que obtuvo una puntuación de 42 , que implicaría un grado de severidad alta en problemas depresivos (el punto de corte para depresión grave es 30 puntos); y también el Cuestionario de Ansiedad Estado-Rasgo (Spielberger, Gorsuch y Lushene, 1970) para determinar los niveles ansiedad iniciales. En Ansiedad-Rasgo obtuvo 23 puntos (percentil 45, un grado de ansiedad medio) lo que implicaría que Milagros no era una persona especialmente propensa a tener ansiedad, sin embargo en Ansiedad-Estado obtuvo 47 puntos (un percentil 96) que sí implicaría la existencia de una situación actual altamente estresante en su vida.

Se le pidieron autorregistros diarios sobre las situaciones que le generaban cualquier tipo de malestar emocional, como situaciones, pensamientos antecedentes, síntomas físicos, conducta motora y qué hacía ella, consecuencias, duración del episodio e intensidad subjetiva valorada (escala de 1 a 10 ). Estos autorregistros de línea base inicial se realizaron durante 3 semanas. En esas anotaciones destacaban aquellos pensamientos relacionados con la pérdida del hijo deseado y emociones fuertes de tipo depresivo y de ansiedad relacionados con la incertidumbre acerca de los resultados de las pruebas de reproducción asistida. Este tipo de reacciones emocionales eran entre 2 y 5 diarias, con una intensidad variable entre 6-10, y también con una duración variable entre pocos minutos y hasta dos horas.

\section{Análisis Funcional}

Se realizaron varios análisis funcionales de los distintos problemas clínicos de Milagros, aunque se presentan aquí sólo las dos hipótesis fundamentales sobre los problemas considerados más prioritarios para el tratamiento. Por un lado, las reacciones emocionales y respuestas de ansiedad (tanto fisiológica como cognitiva) provocadas por las situaciones del tratamiento de reproducción asistida, con un esquema básico de condicionamiento clásico (ver Figura 1); y por otro, la gran cantidad de pensamientos negativos, depresivos y baja autoestima que eran constantes ante todas las situaciones relacionadas con pérdida del hijo, embarazos, niños, etc., con un esquema básico de castigo y extinción (ver Figura 2).

\section{Figura 1}

Hipótesis del Análisis Funcional de los problemas de ansiedad.

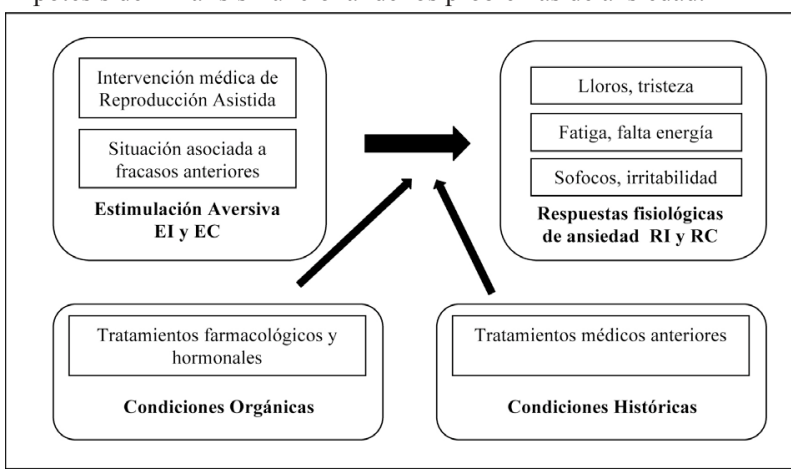

Figura 2

Hipótesis del Análisis Funcional de los problemas depresivos.

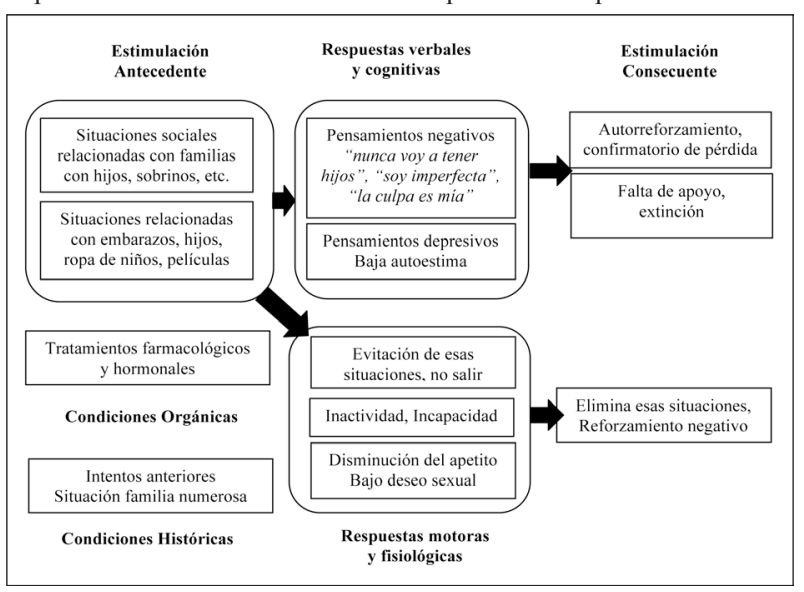


Por otro lado, de forma global también se realizó un análisis funcional de interrelación de los distintos tipos de problemas, que podrían servir de hipótesis general sobre el mantenimiento general de esa situación (ver Figura 3). En esta hipótesis, el tratamiento médico de reproducción asistida serviría como situación desencadenante del resto de problemas, por un lado, como situación estresante o aversiva (por sus propias características supone una situación altamente aversiva para cualquier mujer), que conllevaría gran cantidad de conductas emocionales negativas (no sólo de ansiedad, sino también llanto y tristeza) y también por el efecto fisiológico del tratamiento hormonal (cambios emocionales bruscos, hipersensibilidad, irritabilidad, sofocos). Si tras esa situación ocurría un fracaso unos días después cuando no conseguía los resultados esperados, ello suponía de nuevo una situación aversiva de castigo y una serie de efectos negativos como el aumento progresivo de respuestas emocionales habituales en depresión (llanto, tristeza, desgana, fatiga), cogniciones negativas sobre sí misma y su situación (indefensión, culpabilidad, pensamientos repetitivos sobre el problema, sobregeneralizaciones, atribuciones erróneas, etc.), y conductas depresivas directas tales como inactividad, pasividad o pérdida de actividad sexual. Dada esta situación repetida desde años atrás en varias ocasiones, cualquier estimulación relacionada con este problema podría inducir también la aparición de conductas depresivas, tanto cognitivas como emocionales y, por consiguiente, también la evitación de todas aquellas situaciones que Milagros hubiese relacionado con ello (es decir, salir a la calle y encontrarse con mujeres embarazadas, niños jugando, escaparates con ropa infantil, películas sobre embarazos y niños, etc.) y también todas aquellas interacciones o conversaciones sociales, incluso con su pareja, que pudiesen estar relacionadas con todo el proceso de reproducción asistida y la falta de hijos. De ahí la dificultad para expresar emociones, hablar sinceramente sobre sus problemas o comunicarlos a otras personas. Con lo cual todo el esquema global de comportamientos se mantenía mediante reforzamiento negativo, al mismo tiempo que autorreforzamiento de todas las preocupaciones y pensamientos negativos sobre su situación.

\section{Figura 3}

Hipótesis del Análisis Funcional general relacionando el mantenimiento de los distintos problemas de caso de Milagros.

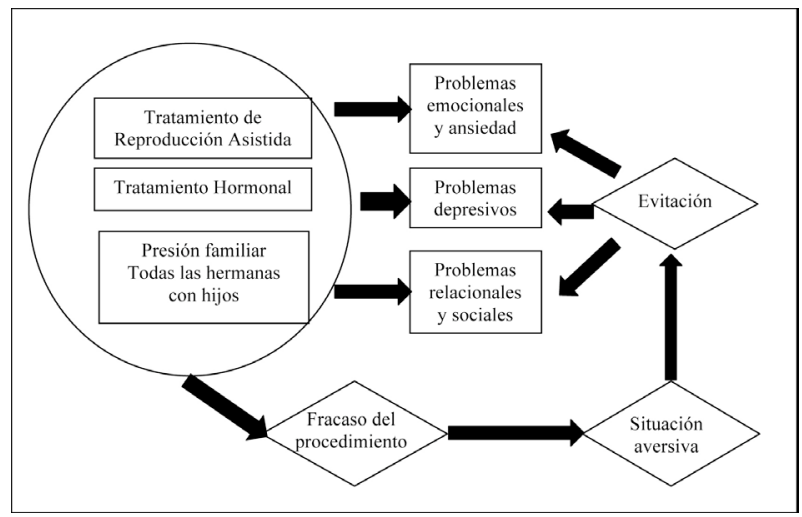

Dadas estas hipótesis funcionales, la planificación del tratamiento se orientó a abordar simultáneamente la problemática en distintos frentes: 1) por un lado, eliminar la evitación y reforzamiento negativo, haciendo que comunicase su situación, expresase sus sentimientos, se comunicase más con su pareja, y al mismo tiempo obtener reforzamiento positivo de tipo social, que normalizase su vida diaria e hiciese menos aversiva toda la situación que estaba viviendo; 2) por otro, disminuir el impacto aversivo de las intervenciones médicas y factores hormonales, mediante relajación y técnicas que disminuyesen las respuestas fisiológicas de ansiedad; y 3) también conseguir mayor cantidad y calidad de reforzamiento positivo de todo tipo respecto a comportamientos y actividades alternativas, para contrarrestar la situación de aislamiento que estaba viviendo, es decir, introducir actividades de terapia de pareja, actividades de ocio, actividades sociales y recreativas, etc., que aumentase todos los aspectos positivos de su vida. En una segunda etapa, 4) se propondrían también como objetivos cambiar el tipo de razonamiento y pensamientos negativos que tenía, mediante técnicas de reestructuración cognitiva y de solución de problemas; 5) y además, mejorar la autoestima y autocontrol de la situación, mediante estrategias de asertividad, escucha, empatía y relaciones sociales más estrechas con el equipo médico. Por último, 6) una etapa de mantenimiento y generalización de los resultados, recordando esas estrategias y su relación cotidiana al tiempo que seguía ocurriendo el proceso médico de reproducción asistida.

\section{Tratamiento}

Siguiendo estas hipótesis y esquema general, se planificaron diversos objetivos terapéuticos progresivos para abordar los problemas de Milagros. Se realizaron un total de 15 sesiones de tratamiento, de 60 minutos de duración, una cada semana.

En una primera fase (sesiones 1 y 2 ) se dieron explicaciones sobre el concepto de ansiedad y estrés, como respuesta de defensa y como reacción a una situación determinada, además de utilizar biblioterapia con artículos y un libro explicando las repercusiones de una intervención médica como la que ella había pasado sobre su estado emocional, también la interacción de esa ansiedad con la propia concepción y con el éxito del tratamiento de reproducción asistida. Al mismo tiempo se le recomendó que buscase más información en Internet en foros específicos y grupos de autoayuda que existen sobre infertilidad.

En la segunda fase (sesión 3) se le fueron sugiriendo actividades alternativas, más gratificantes y distractoras, con objeto de aumentar la cantidad de reforzamiento social que tenía en su vida cotidiana. También se incluyeron tareas para realizar en casa, algunas de ellas para implicarse más en la relación familiar, tales como tareas manuales que le gustaban: coser, crucigramas, leer, escuchar música, baño relajante, y "el día de la pareja", un día a la semana reservado para disfrutar en pareja. Con este mismo objetivo, durante las sesiones se incluían explicaciones para expresar sentimientos, mayor comunicación con la familia y búsqueda de apoyo en su pareja y los familiares. 
En esta tercera fase (sesiones 4 y 5) se incluyó el control de emociones mediante relajación progresiva, relajación abdominal y entrenamiento en visualización de situaciones de relajación, todo ello con objeto de disminuir las respuestas fisiológicas de ansiedad y estrés en su vida cotidiana. Las sesiones 6 a 10 en una tercera fase, se dedicaron a intentar cambiar sus pensamientos negativos y depresivos mediante técnicas de reestructuración cognitiva (errores de pensamiento, pruebas de realidad, sobregeneralización). Se le enseñó a sustituir por pensamientos más racionales y positivos, por ejemplo, "soy una mujer imperfecta" por "soy válida, tenga o no tenga hijos, mi capacidad no depende de eso"; "todos me miran mal" por "mi familia me quiere igual tenga o no tenga hijos"; o "nunca voy a tener hijos" por "es una anticipación, estamos haciendo todo lo que está en nuestras manos"; o "todos tienen hijos menos yo" por "hay mucha gente que no tiene hijos biológicos".

En la cuarta fase (sesiones 11 a 14) se intentó incrementar su percepción de control de la situación y las habilidades asertivas para enfrentarse a la comunicación de sus problemas en todos los aspectos: pareja, familiares y equipo médico. En este caso se le entrenó en técnicas de solución de problemas y toma de decisiones, agendas de autorregistro como recordatorio, expresión de sentimientos y escucha empática, petición de informaciones, petición de cambios, petición de apoyos personales, conversaciones asertivas y "mensajes yo".

Finalmente en la última sesión (sesión 15), se realizó un repaso en general a todas las técnicas y avances conseguidos en las sesiones, y también explicación sobre prevención de recaídas, evaluando los resultados finales con los cuestionarios.

Un mes después se realizó una entrevista de seguimiento, donde se volvieron a aplicar los cuestionarios de evaluación iniciales (BDI y STAI-E), para comprobar el mantenimiento tras ese tiempo. En sucesivas ocasiones posteriores (3, 6 y 12 meses) se realizaron entrevistas telefónicas de seguimiento, donde se le preguntaba revisando los objetivos conseguidos, el estado de la sintomatología que presentaba al inicio y ahora había desaparecido, y el estado subjetivo de bienestar. El objetivo era comprobar el efecto a largo plazo de la terapia realizada y comprobar que se mantenían esos resultados, y que no había problemas de ansiedad o depresión en su vida cotidiana, aunque en este caso no se volvieron a aplicar los cuestionarios.

\section{Resultados}

Los cambios en Milagros fueron evidentes semana tras semana. Progresivamente fueron desapareciendo los problemas de ansiedad, al tiempo que se incrementaron los contactos sociales y las interacciones positivas con su pareja y su familia. Cuando informó a su familia de su situación de infertilidad y los tratamientos de reproducción asistida que estaba haciendo, recibió un gran apoyo de todos ellos no sólo de tipo emocional sino también económico. Al compartir con otras personas a través de Internet sus problemas en foros y grupos de autoayuda sobre infertilidad, comenzó a sentir que no era la única mujer que tenía ese problema. En conjunto, fueron desapareciendo los sentimientos de soledad y aislamiento, ya podía expresar libremente sus emociones y preocupaciones. La comunicación se volvía más fluida con todos los que le rodeaban, mostraba también comportamientos más asertivos para pedir información a los equipos médicos implicados.

Progresivamente fue adquiriendo mayor control sobre su problema, con más información, y fue adoptando un papel más activo en todo el proceso médico, incluso tomando decisiones importantes sobre ello, tales como intentarlo también por la Seguridad Social, plantearse terminar de una vez con los tratamientos médicos, e incluso la posibilidad de adopción.

Todo ello consiguió que desaparecieran tantas emociones y pensamientos negativos que tenía al principio, que viese su problema de una forma más realista y con bastante más calma que en las sesiones iniciales. Aplicaba sistemáticamente el cambio de pensamiento, generando otros más realistas y adecuados a la situación. Con esos cambios ya no evitaba situaciones sociales con niños, escaparates de ropa infantil, o ver embarazadas. Su estado de ánimo fue mejorando y recuperando sus actividades diarias, aunque aún siguiese con tratamientos médicos de reproducción asistida.

Durante este año en que transcurrió la terapia, Milagros continuaba con pruebas médicas para estudiar posibles causas de la infertilidad. Durante ese tiempo realizó dos tratamientos de reproducción asistida, sin éxito. Sin embargo, cuando se realizó el primer seguimiento un mes después, informó que había realizado un último intento mediante donación de ovocitos, con el resultado de un embarazo múltiple, que tras el reposo y control médico adecuado, llegaría a término sin problemas, dando a luz a dos niñas gemelas totalmente sanas. En la Figura 4 puede observarse la disminución en las puntuaciones de los cuestionarios empleados, que pasan en el caso del BDI desde un índice de depresión severa (42 puntos) a problema leve (11 puntos) al final del tratamiento, y ausencia de problemas en el primer mes de seguimiento ( 5 puntos, cuando el punto de corte para depresión leve es 10 puntos). Por su parte, también las puntuaciones del STAI-E disminuyen el grado de ansiedad desde un percentil 96 inicialmente, hasta 50 en el post, y 45 en seguimiento, que implicaban un nivel medio de ansiedad.

\section{Figura 4}

Resultados de la evaluación pre-post-seguimiento en los cuestionarios sobre depresión y ansiedad.

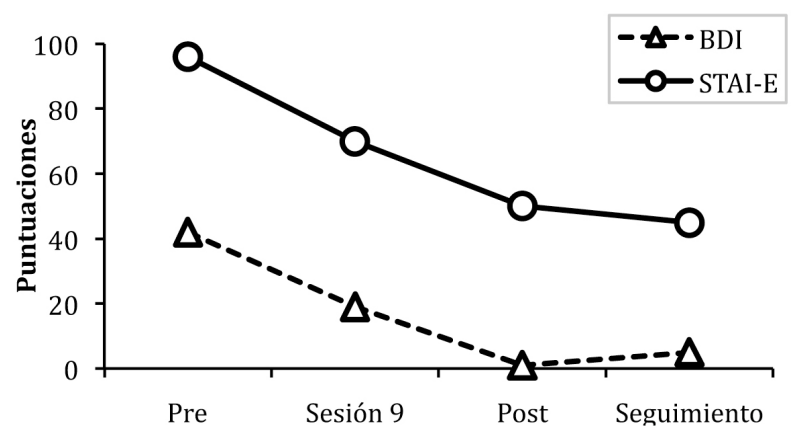


En los seguimientos realizados a los 3, 6 y 12 meses estos resultados se mantuvieron, habían desaparecido los problemas de ansiedad y depresión. En todo caso, un año después Milagros y su pareja tenían las preocupaciones y estrés habituales en el cuidado de dos niñas recién nacidas.

\section{Conclusiones}

Se ha mostrado en este caso clínico la importancia de un tratamiento psicológico individualizado para abordar las consecuencias de una intervención médica en reproducción asistida, que generalmente tiene efectos muy aversivos para las mujeres intervenidas. No se puede afirmar aquí con seguridad que el tratamiento psicológico sea un factor del éxito del tratamiento médico y de que hubiese un embarazo final, tras múltiples intentos fallidos anteriores. Sin embargo, existe literatura especializada que afirma esta interacción entre la situación psicológica y emocional de la mujer y la probabilidad de éxito en intervenciones de este tipo (Boibin, 2003; Domar et al., 1990; Dolz y García, 2002), o bien que esa interacción hace posible múltiples re-intervenciones y que las parejas no desistan en los primeros intentos.

Sin embargo, en un estudio de meta-análisis reciente sobre la relación entre problemas de ansiedad y la fertilidad en procesos de reproducción asistida, que ha recibido también sus críticas (Boivin, Griffitsh y Venetis, 2011a, 2011b; Lynch y Domar, 2011), no puede establecerse una relación significativa entre la disminución en los niveles de ansiedad y un mayor índice de éxitos en los tratamientos de reproducción asistida.

No afirmamos aquí que el tratamiento psicológico, con la consiguiente disminución de los niveles de ansiedad y depresión en esta persona, sea la causa de un embarazo posterior. Lo que sí puede afirmarse con seguridad es que el tratamiento psicológico ha minimizado las consecuencias y efectos aversivos que este proceso conlleva en las personas que pasan por las intervenciones una y otra vez, con fracasos sucesivos (Guerra et al., 2009; Llavona, 2008; Moreno-Rosset et al., 2007; Moreno-Rosset et al., 2009). Se ha mostrado en este caso clínico que, con un tratamiento adecuado, pueden abordarse progresivamente los problemas de ansiedad generados por la operación y el tratamiento hormonal, los problemas depresivos propiciados por toda la situación social y médica asociada, y la gran cantidad de pensamientos negativos y baja autoestima que acompañan a esta situación de infertilidad.

En muchos de esos casos la intervención psicológica podría ayudar no sólo a mejorar la probabilidad de éxito de embarazo, sino también intervenir en el propio proceso ayudando a la paciente, normalizando y disminuyendo el efecto aversivo de todo el contexto hospitalario. Además, el tratamiento psicológico en paralelo al médico, ayudaría a prevenir problemas de ansiedad y depresión muy probables en estas mujeres, cuando se repiten una y otra vez las intervenciones de reproducción asistida. En todo caso, cuando ya se han producido esos efectos, y realmente la mujer pasa por un fuerte estado emocional de ansiedad y depresión, sí que es necesario ese tratamiento para eliminar sus efectos y ayudar a adoptar un punto de vista más realista y quizás otras posibilidades de vida familiar.

\section{Referencias}

1. Antequera, R., Moreno-Rosset, C., Jenaro, C. y Avila, A. (2008). Principales trastornos psicológicos asociados a la infertilidad. Papeles del Psicólogo, 29, 167-175.

2. American Psychiatric Association (2002). DSM-IV-TR Manual de diagnóstico y estadístico de los trastornos mentales. Texto revisado. Barcelona: Masson.

3. Ávila, A. y Moreno-Rosset, C. (2008). La intervención psicológica en infertilidad: Orientaciones para un protocolo de actuación clínica. Papeles del Psicólogo, 29, 186-192.

4. Beck, A.T., Ward, C.H. y Mendelson, M. (1961/1975). BDI Inventory for measuring depression. Archives of General Psychiatry, 4, 561-571. [Versión española Conde, V., y Useros, E. Adaptación castellana de la Escala de Evaluación Conductual para la Depresión de Beck. Revista de Psiquiatría y Psicología Médica, 1975, 12, 217-236].

5. Boivin, J. (2003). A review of psychosocial intervention in infertility. Social Science Medicine, 57, 2325-2341. http:// dx.doi.org/10.1016/S0277-9536(03)00138-2

6. Boivin, J., Griffitsh, E. y Venetis, C.A. (2011a). Emotional distress in infertile women and failure of assisted reproductive technologies: meta-analysis of prospective psychosocial studies. British Medical Journal, 342, 223. Documento on-line consultado el 04-07-11, disponible en http://www.bmj.com/content/342/bmj.d223.full.pdf

7. Boivin, J., Griffitsh, E. y Venetis, C.A. (2011b). The latest word on emotional distress and ART. British Medical Journal, 342, 10-1136. Documento on-line consultado el 04-07-11, disponible en http://www.bmj.com/content/342/ bmj.d223/reply\#bmj_el_260759

8. Castro, C., Borrás, C., Pérez-Pareja, J. y Palmer, A. (2001). Respuestas emocionales en mujeres que se encuentran en tratamiento de Reproducción Asistida, Ansiedad y Estrés, 7, 283-291.

9. Daya, S. (1998). Definitions and factors affecting infertility. En O. Rodríguez-Armas, B. Hedón y S. Daya (Eds). Infertility and Contraception. New York: Parthenon, The International Federation of Fertility Societies.

10. Demyttenaere, K., Nijs, P., Evers-Kiebooms G. y Koninc, K. X. (1991). Coping, ineffectiveness of coping and the psychoendocrinological stress responses during in vitro fertilization, Journal of Psychosomatic Research, 35, 2-3. http://dx.doi.org/10.1016/0022-3999(91)90077-2

11. Dolz, P. y García, A. (2002). Incidencia positiva de los Programas de Apoyo Psicológicos en los Tratamientos de Reproducción Asistida. Revista Iberoamericana de 
Fertilidad y Reproducción Humana. Número especial del 24 Congreso de la Sociedad Española de Fertilidad, 233.

12. Domar, A.D. (1997). Stress and infertility in women. En S.R. Leiblum (Ed). Infertility: Psychological Issues and Counselling Strategies. Canada: John Wiley \& Sons Inc.

13. Domar, A.D., Seibel, M.M. y Benson, H. (1990). The Mind/ Body Program for infertility: A new behavioral treatment approach for women with infertility. Fertility and Sterility, 53, 246-249.

14. Domar, A.D., Clapp, D., Slawsby, E., Kessel, B., Orav, J. y Freizinger, M. (2000). The impact o f group psychological interventions on distress in infertile women. Health Psychology, 19, 568-575. http://dx.doi.org/10.1037/0278$\underline{6133.19 .6 .568}$

15. Domar, A.D., Zuttermeister, P.C. y Friedman, R. (1993). The psychological impact of infertility: a comparison with patients with other medical conditions. Journal of Psychosomatic Obstetrics and Gynaecology, 14-CY, 45-52.

16. Flores, N., Jenaro, C. y Moreno-Rosset, C. (2008). Terapia de pareja en infertilidad. Papeles del Psicólogo, 29, 2005212.

17. Guerra, D. (1998). Cómo afrontar la esterilidad. Barcelona: Planeta.

18. Guerra, D. (2000). Infertilidad y Esterilidad: apoyo e intervención psicológicos. Información Psicológica, 73, 20-29.

19. Guerra, D. y Veiga, A. (1994). Aspectos psicosomáticos en el tratamiento de la esterilidad, en Cuadernos de Medicina Psicosomática, 30, 117-123.

20. Guerra, D., Giménez, V., Baccino, G., Dolz, P., Gil, M.A., Moreno, A., Parra, J., Roca, M., Seijo, M.I. y Tirado, M.M. (2009). Guías de evaluación, consejo, apoyo e intervención psicológica en reproducción asistida (2 $2^{\mathrm{a}}$ parte). Revista Iberoamericana de Fertilidad, 26. Número monográfico.

21. Guerra, D., Llobera, A., Veiga, A y Barri, P. (1998). Morbilidad psiquiátrica en parejas atendidas en Servicios de Infertilidad. Human Reproduction, 13, 1733-1739. http:// dx.doi.org/10.1093/humrep/13.6.1733

22. Instituto Nacional de Estadística (2010). Movimiento natural de la población e indicadores demográficos básicos: Natalidad y Fecundidad. Documento on-line consultado el 7-2-2011 en http://www.ine.es

23. Llavona, L. y Mora, R. (2003). Atención psicológica a las parejas infértiles en tratamiento de reproducción asistida. En J.M. Ortigosa, M.J. Quiles, F.J. y F.J. Médez (eds) Manual de Psicología de la Salud en Niños, Adolescentes y Familia (pp. 287-304). Madrid: Pirámide.

24. Llavona, L.M. (2008). El impacto psicológico de la infertilidad. Papeles del Psicólogo, 29, 158-166.

25. Lynch, C.D. y Domar, A. (2011). Meta-analysis is not the final word on the effect of emotional distress on fertility treatment outcomes. British Medical Journal, 342,
10-1136. Documento on-line consultado el 04-07-11, disponible en http://www.bmj.com/content/342/bmj.d223/ reply\#bmj el 260759

26. Miranda, C., Larrazabal, F. y Laban, P. (1995). Orientación familiar en parejas infértiles. Revista Chilena de Obstetricia y Ginecología, 60, 75-80.

27. Moreno Rosset, C. (2000). Factores psicológicos de la infertilidad. Madrid: Sanz y Torres.

28. Moreno-Rosset, A., Guerra, D., Baccio, G., Jiménez, V., Dolz, P., Tirado, M., Gutiérrez, K. y Gil, M. (2007). Guías de evaluación, consejo, apoyo e intervención psicológica en reproducción asistida. Revista Iberoamericana de Fertilidad, Número monográfico.

29. Moreno-Rosset, C. (2007). Memoria final del proyecto $I+D+I$ Infertilidad en la mujer: Aspectos psicosociales $y$ neurobiológicos. Madrid: Ministerio de Trabajo y Asuntos Sociales.

30. Moreno-Rosset, C., Jenaro, C., Antequera, R. y Gómez, Y. (2009). La psicología de la reproducción: La necesidad del psicólogo en las unidades de reproducción humana. Clínica y Salud: Revista de Psicología Clínica y de la Salud, 20, 79-90.

31. Newman, N.E. y Zouves, C.G. (1991). Emotional experiences of in Vitro Fertilization participants. Journal of Vitro Fertilization and Embryology Transfer, 8, 322-328. http:// dx.doi.org/10.1007/BF01133022

32. Newton, C.R., Hearn, M.T. y Yuzpe, A.A. (1990). Psychological assessment and follow-up after in vitro fertilization: assessing the impact of failure. Fertility and Sterility, 54, 879-886.

33. Palacios, E., Jadresic, E., Palacios, B., Miranda, C. y Domínguez, R. (2002). Estudio descriptivo de los aspectos emocionales asociados a la infertilidad. Revista, Chilena de Obstetricia y Ginecología, 67, 19-24.

34. Robles, H. y Peralta, M.I. (2006). Programa para el control del estrés. Madrid: Pirámide.

35. Sander, K.A. y Bruce, N.W. (1999). Psychological stress and treatment outcome following assisted reproductive technology. Human Reproduction, 14, 1656-62. http:// dx.doi.org/10.1093/humrep/14.6.1656

36. Seger, L. (2003). El impacto emocional de la infertilidad en las fases de la reproducción asistida. Psicología Conductual: Revista Internacional de Psicología Clínica y de la Salud, 1, 95-113.

37. Seibel, M.M. y Taymor, M.L. (1982). Emotional aspects of infertility. Fertility and Sterility, 37, 137-145.

38. Spielberger, C.D., Gorsuch, R.L. y Lushene, R.E. (1970). STAI Cuestionario de Ansiedad Estado/Rasgo. Madrid: Tea Ediciones.

39. Tarlatzis, I., Tarlatzis, I., Diakogiannis, J., Bontis, S., Lagos, D., Gavrillidou, D. y Mantelenakis, S. (1993). Psychosocial impacts of infertility on Greek couples. Human Reproduction, 8, 396-401. 
40. Tuschen, C.B., Florín, L., Krause, W. y Pook, M. (1999). Cognitive-Behavioral Therapy for Idiopathic Infertile Couples. Psychotherapy and Psychosomatic, 68, 15-21. http://dx.doi.org/10.1159/000012305

41. World Health Organization (2003). Progress Report in Reproductive Health Research, 23. Geneva, Switzeland.

Fecha de recepción: 26 de marzo de 2011

Fecha de recepción de la versión modificada: 1 de septiembre de 2011

Fecha de aceptación: 19 de septiembre de 2011 